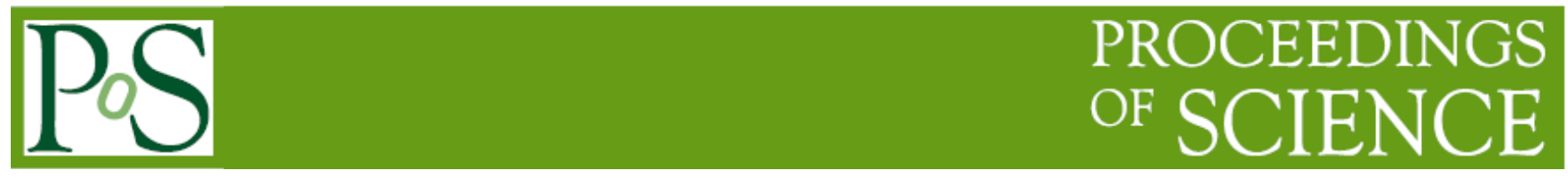

\title{
Effect of solar poloidal magnetic field reversal on tri-diurnal anisotropy of cosmic ray intensity on quiet days
}

\author{
M.K. Richharia \\ Department of Physics \\ Govt. Model Science College (Autonomous) Jabalpur (M.P.) India \\ (E-mail: mkrichharia@yahoo.com)
}

Presently at: Govt. R.D.College Mandla (M.P.) India

The tri-diurnal and quart diurnal anisotropy of cosmic ray intensity have been investigated during the solar cycle 21-22 using the neutron monitor data recorded at different latitudes on sixty geo magnetically quiet days in a year. It has been observed that in spite of the abrupt changes in the amplitude and phase of tri-diurnal and quart diurnal anisotropy in cosmic ray intensity, the phase of tri-diurnal anisotropy shifted to earlier/later hours during the reversal period 1990-91 and 1979-80 showing the dependence on the polarity of solar magnetic field, which is attributed to drift effect at mid latitude neutron monitoring stations. Further, no systematic change have been noticed in the amplitude and phase of quart-diurnal anisotropy of daily variation in quiet days.

Key words: Cosmic Ray/Gamogenetically quiet days/ Higher harmonics. 


\section{Introduction}

The anisotropic variation of galactic cosmic rays and their characteristics are studied through the diurnal, semi-diurnal, Tri-diurnal component mainly and the level of the isotropic intensity collectively provides fingerprint for identifying the modulating process and the electromagnetic state of interplanetary space in the neighborhood of the Earth. Many workers have attempted to derive relationship between the mean daily variation and the level of solar and geomagnetic activity. [1] The spatial anisotropy of the galactic cosmic ray intensity in the interplanetary space manifests itself as daily variation with a period of 24 hours (and its higher harmonics) due to the rotation of the Earth in the course of a day. The power Spectrum analysis as well as the Fourier analysis of the long term data of the 24-hour values of comic rays (CR) intensity observed by Earth based detectors have provided daily variation of extraterrestrial origin [2-4]. However, the amplitude of the fourth harmonics is still controversial [5-7]. Moreover, it has been observed that the amplitude and phase of tri diurnal anisotropy of CR intensity on quiet days vary considerably from one period to another. On the long term behaviour of the first three harmonics showed that high degree of year to year variability exists, a trend with solar activity was evident. The studies of the higher harmonics in the daily variation of cosmic ray provided valuable information as to the nature of the cosmic ray modulation in the heliosphere.

\section{Analysis of the data}

Solar daily variation has been studied in terms of helio-magnetic activity. A new concept of data analysis has been introduced for studying the long/short term daily variation in CR intensity recorded with neutron monitors. Fourier technique has been applied on different types of group of days chosen according to their different geomagnetic condition.

1. All days: This means all the $365 / 366$ days in year. Thus, these days are termed as AD. Of course ignoring the days with abrupt changes.

2. Quiet days: Those days on which the transient magnetic variation are regular and smooth are said to be magnetically quiet or Q days. The criteria is based upon Ap and Kp values. There are two types of days.

(i) 60 Quiet days: According to solar geophysical data (SGD) lowest mean order number are the five quietest days in a month. Thus, $60 \mathrm{Q}$ days in a year; termed as 60 QD.

(ii) 120 Quiet days: First ten quiet days in a month. Thus, 120 Q Days in a year; termed as 120 Quiet days. 
The pressure corrected hourly CR intensity data (corrected for meteorological effects) on geomagnetically five quietest days (QD) in every month for Deep River (Lat: $46.06^{\circ} \mathrm{N}$; Cutoff rigidity; $1.02 \mathrm{GV}$; Longitude; $282.5^{\circ} \mathrm{E}$; Altitude; $145 \mathrm{~m}$ ) and Goose Bay (Lat: $53.33^{\circ} \mathrm{N}$; Cutoff rigidity; $0.52 \mathrm{GV}$; Longitude; $299.58^{\circ} \mathrm{E}$; Altitude; 46m) neutron monitoring stations for the period 1978-94, have been used in Fourier analysis. After applying the trend corrections, such a set of data have been subjected to Harmonic analysis for each day [8]. The average values of the amplitude and phase (local tine of the station) of the third (tri diurnal) harmonics on yearly basis have been obtained. According to solar geophysical data five quietest days are selected in a month; thus 60 quietest days are obtained in a year. These days are called international quiet days (QD). The days with extra ordinary large amplitude, if any, have not been considered. Further, the variation in the tri-diumal anisotropy with the reversal of polarity of solar magnetic field (PSMF) on 60 QD has been also investigated. Also all those days are discarded having more than three continuous hourly data missing.

\section{Results and discussion}

The yearly average amplitude and phase of the third harmonics of daily variation along with PSMF in the northern and southern hemisphere of the Sun for Deep River and Goose Bay Neutron Monitoring Stations have been plotted in Fig. 1 \& 2 during the period 1978-94 on quiet days. It is quite apparent from Fig. 1 at Deep River Neutron Monitoring Station, the amplitude of third harmonics of daily variation has quite abruptly increased during the years 1980 and 1985. The likely one of the cause for such type of variation could be the changing of geomagnetic threshold cut off rigidly from $1.02 \mathrm{GV}$ to $1.15 \mathrm{GV}$ in 1980 and from 1.02 GV to $1.12 \mathrm{GV}$ in 1985 respectively [9-11] as it has been discussed in the case of change of diurnal anisotropy of cosmic ray intensity on QD [12], Therefore, these type of variation in the amplitude of the tri-diumal anisotropy on QD may be also attributed to the change in the rigidity spectrum. The amplitude of tri-diumal anisotropy on QD has shown as exceptionally small value during 1987 and 1994, which is a period of minimum solar activity The amplitude of third harmonics of daily variation on QD is observed to be significantly low during 1981 as well as in 1990, which coincides with phase reversal of the solar poloidal magnetic field. Further, during the year 1985 and 1986, the year of minimum solar activity, the amplitude of third harmonics on QD significantly high, which support the earlier finding. [13] 


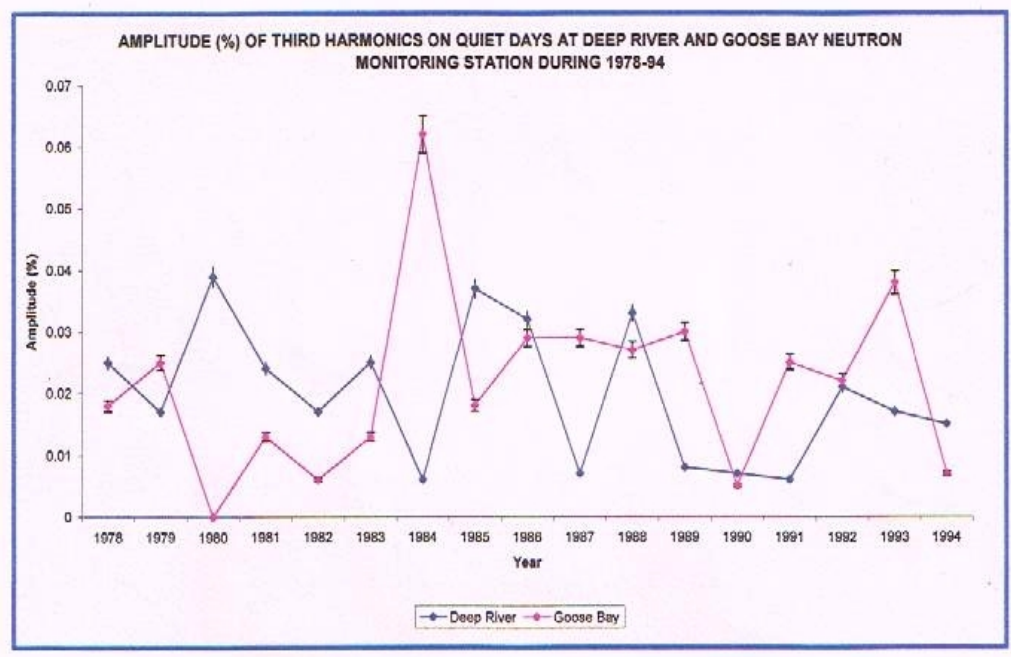

Figure 1

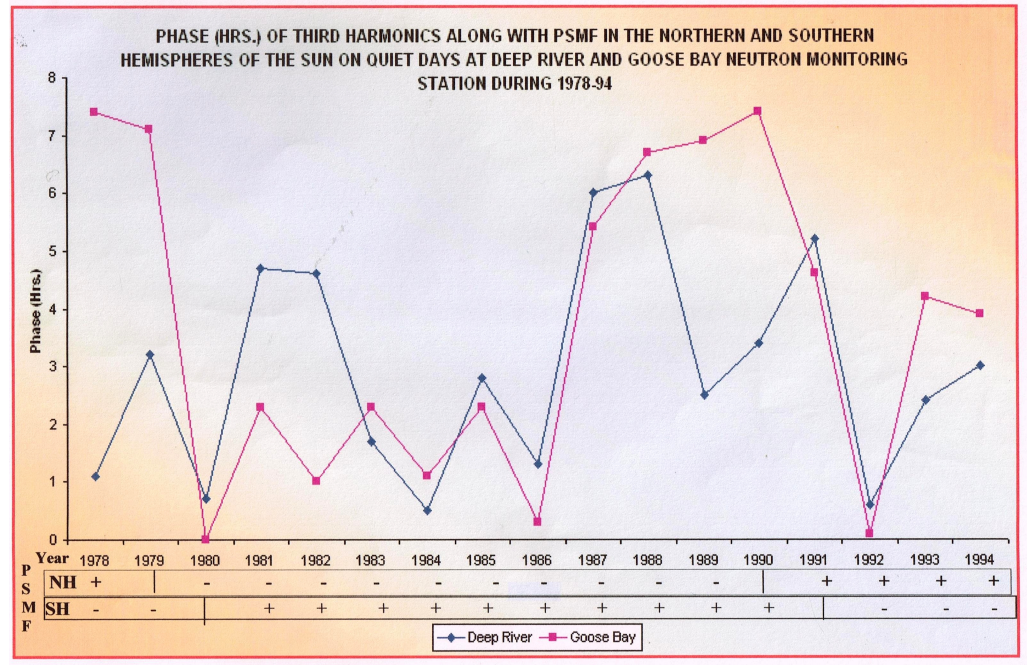

Figure 2

It is apparent from Fig.l that there is no systematic variation is observed in the amplitude of the third harmonics of daily variation at Goose Bay Neutron Monitoring Stations. However, some of the significant observations are such as the amplitude is decreased during the year 1990, which coincides with phase reversal of the solar poloidal field. It is observed that the amplitude is statistically nearly constant high value during the years 1986- 88. The amplitude of the Tri-diurnal anisotropy of CR intensity has been observed almost equal during the two consecutive solar maximum activity periods i.e., (year 1979 and 1991) of solar cycles - 21 and 22 supporting 11- year variation in the Tri-diurnal anisotropy of CR intensity on quiet days. Further, the amplitude of third harmonics during the years, 1982 and 1994 (Difference at 11 year) having the same value, which is again confirm the 11 year type variation in third harmonics of daily variation [13]. 
The nearly equal values of the amplitude of third harmonics has been observed during the year 86, 88, 90, 92 at mid latitude neutron monitoring stations.

It is also observed from Fig. 2 at mid latitude neutron monitoring stations, that there is no systematic change in the phase of third harmonics of daily variation in cosmic ray intensity on quiet days. However, a slight change in the tri-diurnal phase is observed, when the polarity of solar magnetic field reversed its polarity during the periods 1979-80 and 199091. It shows that the phase of tri-diurnal anisotropy on quiet days has nearly the same value at both sides of reversal period. Whereas in both the cases during the succeeding years, i.e., 1980-81 and 1991-92, the change in the phase of tri-diurnal anisotropy of CR intensity has been found quite significant $[14,15]$. It is clear that there exist a 11 year type of variation in third harmonics of CR intensity on QD due to the variation of solar activity [16]. The polarity dependence of the phase shift change has been interpreted as a result due to the change of CR density distribution in space caused by the difference of CR drift motion in the positive and negative polarity state. The existence of polarity dependence in the tri-diurnal variation may be defined as the state is positive, when the magnetic field is away from the sun at the north pole and toward the Sun at the south pole, while it is called negative, when the polar magnetic field are reversed [17].

The yearly average amplitude and phase of fourth harmonics of daily variation on quiet days for Deep River and Goose Bay Neutron Monitoring station have been plotted in Fig. 3 \& Fig. 4 during the period 1978-94. It is quite apparent from the Fig. 3 that there is no systematic change noticed in the amplitude of the fourth harmonics of daily variation on quiet days, the amplitude during the year 1979 and 1985 has quite abruptly increased. Further the year 1987, the amplitude has small value, which is a period of minimum solar activity. The amplitude during the years 1980 and 1991 has been observed same value, which is period of high solar activity in the solar cycle 21 and 22. This confirm the 11 year type variation also occur in the fourth harmonics of daily variation at Deep River Neutron monitoring station. Furthermore, it is quite apparent from Fig. 4, that there is no systematic change in the phase of fourth harmonics, the phase in the year 1981 and 1992 has occurred in the same direction, which may be indicate likely 11 year type variation at Deep River Neutron monitoring station [16].

It is quite apparent from the Fig. 3, that there is no systematic change in the amplitude of fourth harmonics of daily variation on quiet days. Likewise, it is also quite apparent from Fig. 4 that there is no systematic variation occurred in the phase of fourth harmonics of daily variation on quiet days. The phase of fourth harmonics during the interval 1982-85 shifted to early hours, but during the interval 1985-87 it is shifted to later hours at Goose Bay neutron monitoring station. 


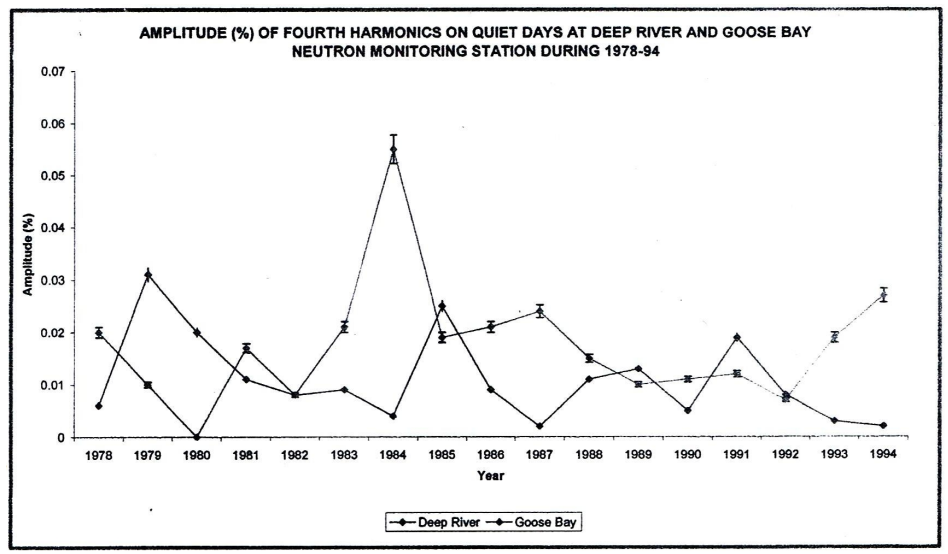

Figure 3

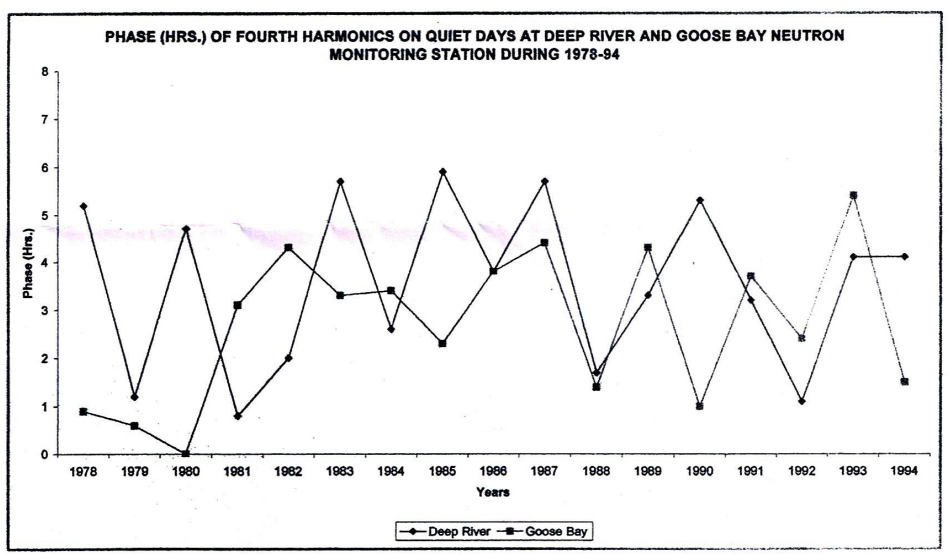

Figure 4

\section{Acknowledgments}

The authors are indebtend to various experimental groups; in particular, Profs. M.

Bercovitch, K. Nagashima and Miss Aoi Inoue for providing neutron monitor data.

\section{References}

[1] D. Venkatesan and Badruddin, Space Science Rev., 52, 121 (1990) and reference there in.

[2] Fujii, A., Nagashima, K., Fujimoto, K., Ueno, H. And Kondo, I. 1971, 12th ICRC, Hobart Tasmania, 2, 666.

[3] Ahluwalia, H. S. and Singh, S. 1973a, Proc. $13^{\text {th }}$ Int. Cosmic Ray Conf., Australia, 2:948.

[4] Ahluwalia, H. S. and Singh, S. 1973b, Proc. $13^{\text {th }}$ Int. Cosmic Ray Conf., Australia, 5:3129. 
Effect of solar poloidal magnetic field reversal on tri-diurnal anisotropy of cosmic ray intensity on quiet days M.K. Richharia

[5] Pomerantz, M. A. and Duggal, S. P. 1971, Space Sci. Rev., 12, 75.

[6] Rao, U. R. 1972, Space Sci. Rev., 12, 719.

[7] Agrawal, S. P. 1981, Journal Geophys. Res., 86: 10115.

[8] Kumar, S., Agrawal, R., Mishra, R. And Dubey, S. K. 2002, Bull Astronomical Soc. India, 30, 451.

[9] Shea, M. A. and Smart, D. F. 1983, 18th Int. Cosmic Ray Conf. Bangalore, 3:411.

[10] Smart, D. F. and Shea, M. A. 1987, 20th Int. Cosmic Ray Conf. Moscow, 4: 204.

[11] Shea, M. A. and Smart, D. F. 2001, 27th Int. Cosmic Ray Conf. Hemberg, 3: 4063.

[12] Kumar, S. Gulati, U., Khare, D. and Richharia, M. K. 1993, Bull Astronomical Soc. India, 21:395.

[13] Kumar, S., Richharia, M. K, Chauhan, M.L., Gulati, U., Khare, D. K. And Shrivastava, S. K. 1995, 24th Int. Cosmic Ray Conf. Italy, 4: 623.

[14] Richharia, M.K., 2007, Pramana, J. Phy., Vol. 68, 6.

[15] Richharia, M.K. 2011, 32 ${ }^{\text {nd }}$ International Cosmic Ray Conference, Beijing China, 11, S.H. 3:4, 127.

[16] Richharia, M.K. 2015, International Journal of Astrophysics and Space Science, Vol. 3, No. 2-1, pp4-6.

[17] Richharia, M.K. 2015, International Journal of Astrophysics and Space Science, Vol. 3, No. 2-1, pp 1-3. 